\title{
Short-Period and Long-Period Effects of Weak Gravitational Waves
}

\author{
M. H. A. Youssef \\ Astronomy Department, Faculty of Science, Cairo University, Giza, Egypt \\ Email: mhyoussef@sci.cu.edu.eg
}

How to cite this paper: Youssef, M.H.A. (2017) Short-Period and Long-Period Effects of Weak Gravitational Waves. International Journal of Astronomy and Astrophysics, 7, 230-237.

https://doi.org/10.4236/ijaa.2017.73019

Received: August 31, 2017

Accepted: September 25, 2017

Published: September 28, 2017

Copyright $\odot 2017$ by author and Scientific Research Publishing Inc. This work is licensed under the Creative Commons Attribution International License (CC BY 4.0).

http://creativecommons.org/licenses/by/4.0/

\section{(c) (i) Open Access}

\begin{abstract}
We compute the long-term orbital variation of a test particle orbiting a central body acted upon by normal incident of plane gravitational wave. We use the tools of celestial mechanics to give the first order solution of canonical equations of long-period and short-period terms of the perturbed Hamiltonian of gravitational waves. We consider normal incident of plane gravitational wave and characteristic size of bound-two body system (earth's satellite or planet) is much smaller than the wavelength of the wave and the wave's frequency $n_{w}$ is much smaller than the particle's orbital $n_{p}$. We construct the Hamiltonian of the gravitational waves in terms of the canonical variables $(l, g, h, L, G, H)$ and we solve the canonical equations numerically using Runge-Kutta fourth order method using language MATHEMATICA V10. Taking Jupiter as practical example we found that there are long period perturbations on $\omega, \Omega$ and $i$ and not changing with revolution and the short period perturbations on $a, e$ and $M$ changing with revolution during the interval of time $\left(t-t_{0}\right)$ which is changing from $0 \rightarrow 4 \pi$.
\end{abstract}

\section{Keywords}

Gravitational Waves, General Relativity, Celestial Mechanics, Perturbation, Orbital Mechanics

\section{Introduction}

The tremendous penetrating capacity of gravitational waves is a reason why it is so difficult to detect them. Another reason is the very low intensities by which they are emitted. A great number of theoreticians and experimentalists work on gravitational radiation not only because of its important applications but also because of its fundamental role in science. In the domain of general relativity, 
the particular solution of the field equations leading to the wave solution is usually preferred in the domain of the post-Newtonian (or weak field) approximation. The reason is that the wave contributes to its own source, besides the effect of the relatively strong field of source itself; greatly complicate the detection of the wave close to its source. Hence it is better to study the simple properties of the waves by looking far into the wave zone where the fields are weak. The effects of incident plane gravitational waves on the orbital motion of gravitational bound two-body system were investigated by several authors with different approaches and various features of both orbits and the waves [1]-[9]. In this work we treat the effect of GW on the orbital motion by semi-analytical treatment and we determine the perturbation on the orbital elements using new approach differ than the previous works. We construct the Hamiltonian of GW and solve the canonical equations in terms of the Delaunay elements $(l, g, h, L, G, H)$, using the numerical method of Runge-Kutta and algebraic manipulations programs MATHEMATICA V10. The resulting theory includes short-period as long-period for practical application on planet Jupiter.

\section{Hamiltonian of GW}

According to the weak field approximation of general relativity, there exist in empty space gravitational waves generated by different sources far in the universe, and propagated with velocity $c$. The field equations for empty space in the linear weak field approximation

$$
\square^{2} h_{i k}=0
$$

Has the plane wave as the simplest solution. $\square^{2}$ is d'Alembertian operator is given by

$$
\square^{2}=\frac{\partial^{2}}{\partial x_{i}^{2}}-\frac{1}{c^{2}} \frac{\partial^{2}}{\partial t^{2}}(i=1,2,3)
$$

With the $Z$ axis chosen in the direction of propagation $h_{i k}, \square^{2}$ becomes

$$
\square^{2}=\frac{\partial^{2}}{\partial z^{2}}-\frac{1}{c^{2}} \frac{\partial^{2}}{\partial t^{2}}
$$

and then the partial derivatives of $h_{i k}$ with respect to $x$ and $y$ vanish. Thus a plane gravitational wave is determined by two quantities $h_{12}$ and $h_{11}=-h_{22}$ in other words, gravitational waves are transverse waves whose polarization is determined by a symmetric tensor of the second rank in the XY plane, the sum of whose diagonal terms $h_{11}+h_{22}$ is zero. So the plane wave can be written a the sum of two components

$$
\begin{aligned}
& h_{11}=h_{+} \cos \left(n_{w} t+\alpha_{1}\right) \\
& h_{12}=h_{\times} \cos \left(n_{w} t+\alpha_{2}\right)
\end{aligned}
$$

where $n_{w}$ is the frequency of the wave, $\alpha_{1}$ and $\alpha_{2}$ are the phase difference, $h_{+}$and $h_{x}$ are the amplitude of the wave in the two orthogonal directions in the transverse plane. From the equation of geodesic deviation we obtain the ac- 
celeration components of GW as in [10].

$$
\frac{\mathrm{d}^{2} n^{i}}{\mathrm{~d} t^{2}}=\frac{1}{2} \frac{\partial^{2} h_{i k}}{\partial t^{2}} n^{k}
$$

From which we can obtained the potential like function $U$ of GW in terms of the orbital elements

$$
U=\frac{1}{2} h_{1}\left(y^{2}-x^{2}\right)-h_{2} x y
$$

where $h_{1}=\frac{1}{2} \frac{\partial^{2} h_{11}}{\partial t^{2}}$ and $h_{2}=\frac{1}{2} \frac{\partial^{2} h_{12}}{\partial t^{2}}$, and we have

$$
\begin{aligned}
& x=r(\cos \Omega \cos (f+\omega)-\cos i \sin \Omega \sin (f+\omega)) \\
& y=r(\sin \Omega \cos (f+\omega)+\cos i \cos \Omega \sin (f+\omega))
\end{aligned}
$$

where $i, \Omega, \omega$ and $f$ are the inclination, longitude of node, argument of perigee and the true anomaly of an orbit respectively. Substituting Equations ((1), (2), (5) and (6)) into Equation (4) we obtain

$$
\begin{aligned}
U= & \epsilon r^{2}\left\{\frac { s ^ { 2 } } { 8 } \left[\cos \left(2 \Omega+n_{w} t+\alpha_{1}\right)-\cos \left(2 \Omega-n_{w} t-\alpha_{1}\right)\right.\right. \\
& \left.-A_{g}\left[\sin \left(2 \Omega+n_{w} t+\alpha_{2}\right)+\sin \left(2 \Omega-n_{w} t-\alpha_{2}\right)\right]\right] \\
& +\left(\frac{c^{2}}{16}+\frac{c}{8}-\frac{1}{16}\right)\left[\cos \left(2 f+2 \omega+2 \Omega+n_{w} t+\alpha_{1}\right)+\cos \left(2 f+2 \omega+2 \Omega-n_{w} t-\alpha_{1}\right)\right. \\
& -A_{g}\left[\sin \left(2 f+2 \omega+2 \Omega+n_{w} t+\alpha_{2}\right)+\sin \left(2 f+2 \omega+2 \Omega-n_{w} t-\alpha_{2}\right)\right. \\
& \left.\left.+\sin \left(2 f+2 \omega-2 \Omega+n_{w} t+\alpha_{2}\right)+\sin \left(2 f+2 \omega-2 \Omega-n_{w} t-\alpha_{2}\right)\right]\right] \\
& \left.+\left(\frac{c^{2}}{16}-\frac{c}{8}+\frac{1}{16}\right)\left[\cos \left(2 f+2 \omega-2 \Omega+n_{w} t+\alpha_{1}\right)+\cos \left(2 f+2 \omega-2 \Omega-n_{w} t-\alpha_{1}\right)\right]\right\}
\end{aligned}
$$

where

$$
c=\cos i, s=\sin i, \epsilon=\frac{1}{2} n_{w}^{2} h_{\times} \text {and } A_{g}=-\frac{h_{\times}}{h_{+}}
$$

The Hamiltonian will be in the form

$$
H=H_{0}+\epsilon H_{1}
$$

$H_{0}$ is the unperturbed Hamiltonian given by

$$
H_{0}=-\frac{\mu^{2}}{2 L^{2}}
$$

$\mu=G M$ the gravitational constant and $L=\sqrt{\mu a}$. The perturbed Hamiltonian $H_{1}$ due to the gravitational wave is given by

$$
\begin{aligned}
H_{1}= & \frac{L^{4}}{\mu^{2}} \frac{r^{2}}{a^{2}}\left\{\frac { s ^ { 2 } } { 8 } \left[\cos \left(2 \Omega+n_{w} t+\alpha_{1}\right)-\cos \left(2 \Omega-n_{w} t-\alpha_{1}\right)\right.\right. \\
& \left.-A_{g}\left[\sin \left(2 \Omega+n_{w} t+\alpha_{2}\right)+\sin \left(2 \Omega-n_{w} t-\alpha_{2}\right)\right]\right] \\
& +\left(\frac{c^{2}}{16}+\frac{c}{8}-\frac{1}{16}\right)\left[\cos \left(2 f+2 \omega+2 \Omega+n_{w} t+\alpha_{1}\right)+\cos \left(2 f+2 \omega+2 \Omega-n_{w} t-\alpha_{1}\right)\right.
\end{aligned}
$$




$$
\begin{aligned}
& -A_{g}\left[\sin \left(2 f+2 \omega+2 \Omega+n_{w} t+\alpha_{2}\right)+\sin \left(2 f+2 \omega+2 \Omega-n_{w} t-\alpha_{2}\right)\right. \\
& \left.\left.+\sin \left(2 f+2 \omega-2 \Omega+n_{w} t+\alpha_{2}\right)+\sin \left(2 f+2 \omega-2 \Omega-n_{w} t-\alpha_{2}\right)\right]\right] \\
& \left.+\left(\frac{c^{2}}{16}-\frac{c}{8}+\frac{1}{16}\right)\left[\cos \left(2 f+2 \omega-2 \Omega+n_{w} t+\alpha_{1}\right)+\cos \left(2 f+2 \omega-2 \Omega-n_{w} t-\alpha_{1}\right)\right]\right\}
\end{aligned}
$$

\section{Equations of Motion}

The Delaunay canonical variables $(l, g, h, L, G, H)$ are defined in terms of the Keplerian elements $(a, e, i, \Omega, \omega, M)$ by

$$
\begin{array}{lll}
l=M=n(t-\tau) & ; L=\sqrt{\mu a} \\
g=\omega ; & G=\sqrt{\mu a}\left(1-e^{2}\right) \\
h=\Omega ; & H=G \cos i
\end{array}
$$

The considered systems of differential equations in terms of canonical elements under the effect of GW are

$$
\frac{\mathrm{d} L_{i}}{\mathrm{~d} t}=-\frac{\partial H}{\partial l_{i}} ; \frac{\mathrm{d} l_{i}}{\mathrm{~d} t}=\frac{\partial H}{\partial L_{i}}(i=1,2,3,4)
$$

Then

$$
\begin{gathered}
l^{\cdot}=\frac{\partial H}{\partial L} ; \quad g^{\cdot}=\frac{\partial H_{1}}{\partial G} ; \quad h^{\cdot}=\frac{\partial H_{1}}{\partial H} \\
L^{\cdot}=-\frac{\partial H_{1}}{\partial M} ; \quad G^{\cdot}=-\frac{\partial H_{1}}{\partial g} ; \quad H^{\cdot}=-\frac{\partial H_{1}}{\partial h}
\end{gathered}
$$

Using Equations ((11), thus (13) and (14)) yield

$$
\begin{gathered}
a^{\cdot}=2 \sqrt{\frac{a}{\mu}} L^{\cdot} \\
e^{\cdot}=\frac{\left(1-e^{2}\right)}{n a^{2} e} L^{\cdot}-\frac{\sqrt{\left(1-e^{2}\right)}}{n a^{2} e} G^{\cdot} \\
\ddot{I}^{\prime}=\frac{\cot i}{n a^{2} \sqrt{\left(1-e^{2}\right)}} G^{\cdot}-\frac{\csc i}{n a^{2} \sqrt{\left(1-e^{2}\right)}} H^{\cdot} \\
\Omega^{\cdot}=\frac{\partial H_{1}}{\partial H}=\frac{\partial H_{1}}{\partial i} \frac{\partial i}{\partial H_{1}} \frac{\partial H_{1}}{\partial G^{*}} \frac{\partial e}{\partial G}+\frac{\partial H_{1}}{\partial i} \frac{\partial i}{\partial G} \\
M^{\bullet}=\frac{\partial H_{0}}{\partial L}+\frac{\partial H_{1}}{\partial L}=\frac{\partial H_{0}}{\partial L}+\frac{\partial H_{1}}{\partial a} \frac{\partial a}{\partial L}+\frac{\partial H_{1}}{\partial e} \frac{\partial e}{\partial L}
\end{gathered}
$$

We solve this equations numerically using Runge-Kutta fourth order method, the mathematical program written by language of MATHEMATIC V10.

\section{Solution and Results}

We expand the trigonometric functions of the true anomaly $\frac{r^{2}}{a^{2}}, \frac{r^{2}}{a^{2}} \cos 2 f$ 
and $\frac{r^{2}}{a^{2}} \sin 2 f$ of Hamiltonian (10) in series of the trigonometric functions of mean anomaly $M$ up to order three in the eccentricity [11]. Changing the independent variable from the time $t$ to the mean anomaly $M$, substituting the Hamiltonian into the equations of motion and solving the equations numerically to obtain the first order perturbation of GW on Jupiter. Table 1 represents the orbital elements of Jupiter in the epoch 2000, considering Bursts of GW with frequency $56 \times 10^{-6} \mathrm{~Hz}$ and amplitude $15 \times 10^{-22} \cdot \alpha_{1}=0$ and $\alpha_{2}=\frac{\pi}{2}$. The variations of Jupiter's elements due to the considering effect of gravitational waves are presented in Table 2, during the interval between zero and $4 \pi$ as in Figure 1.

Table 1. The orbital elements of Jupiter Reference date 12.00 UT 1 Jan. 2000.

\begin{tabular}{cc}
\hline Elements of the Planet & Jupiter \\
\hline The Semi-major in km $(a)$ & $778 \times 10^{6}$ \\
The eccentricity $(e)$ & 0.048 \\
Inclination in degree $(i)$ & 1.30536 \\
Longitude of node in degree $\Omega$ & 100 \\
Longitude of perihelion in degree $\omega$ & 13.678 \\
Mean daily motion in degree/day $n$ & 0.0831
\end{tabular}

Table 2. The perturbed orbital elements of Jupiter due the incident of GW.

\begin{tabular}{ccccccc}
\hline interval & $a \mathrm{~km}$ & $e$ & $I$ radian & $\Omega$ radian & $\Omega$ radian & $M$ radian \\
\hline 1 & $7.7835 \times 10^{13}$ & 0.0481105 & 0.0227591 & 1.75231 & 4.78744 & 0.138604 \\
2 & $7.78341 \times 10^{13}$ & 0.0480292 & 0.0227591 & 1.75231 & 4.78744 & 0.238604 \\
3 & $7.78334 \times 10^{13}$ & 0.0479074 & 0.0227591 & 1.75231 & 4.78744 & 0.338604 \\
4 & $7.78318 \times 10^{13}$ & 0.0478299 & 0.0227591 & 1.75231 & 4.78744 & 0.438604 \\
5 & $7.78308 \times 10^{13}$ & 0.0477027 & 0.0227591 & 1.75231 & 4.78744 & 0.538604 \\
6 & $7.7831 \times 10^{13}$ & 0.0476119 & 0.0227591 & 1.75231 & 4.78744 & 0.638604 \\
7 & $7.78311 \times 10^{13}$ & 0.0475403 & 0.0227591 & 1.75231 & 4.78744 & 0.738604
\end{tabular}

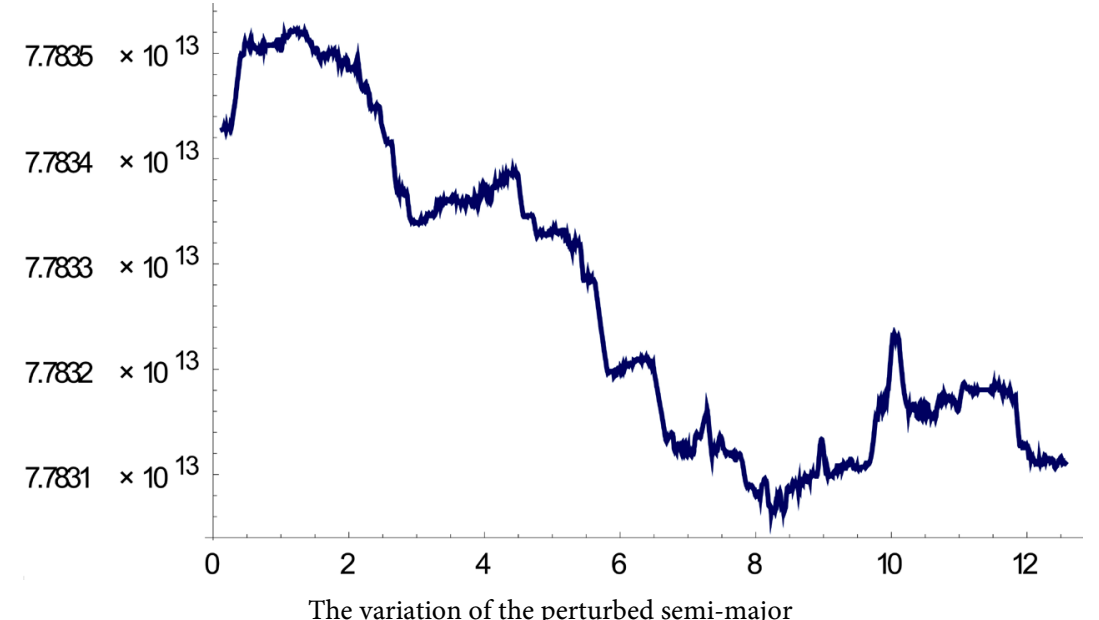

$\mathrm{X}$-axis represents the variation of time from 0 to $4 \pi$ in degree and $\mathrm{Y}$-axis represents $\delta \mathrm{a}$ in radian. 


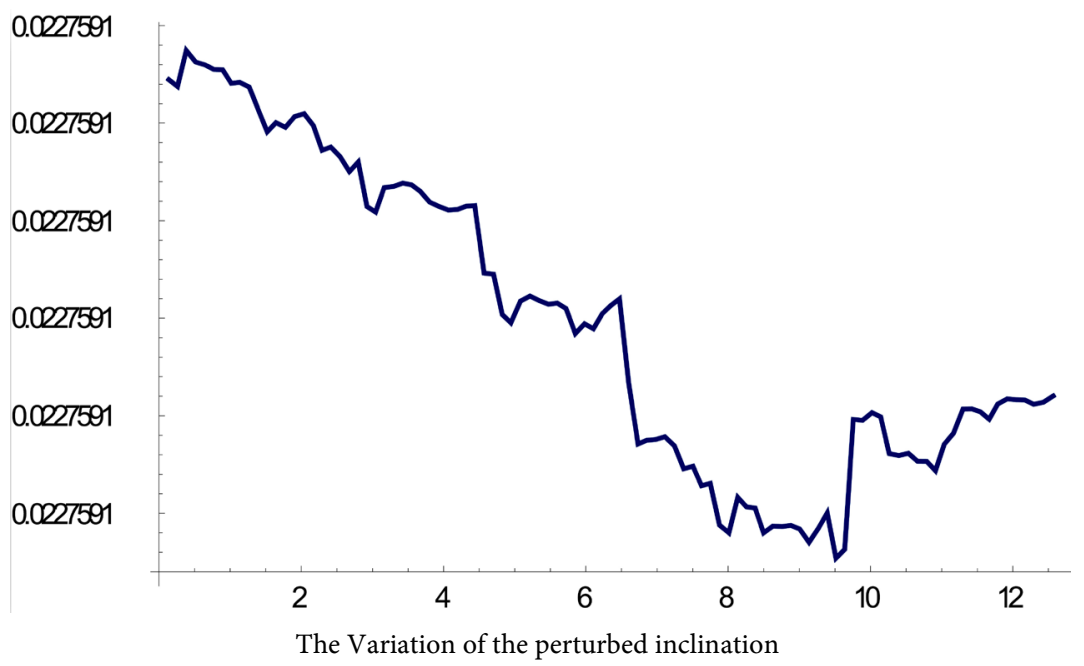

$\mathrm{X}$-axis represents the variation of time from 0 to $4 \pi$ in degree and $\mathrm{Y}$-axis represents $\delta \mathrm{i}$ in radian

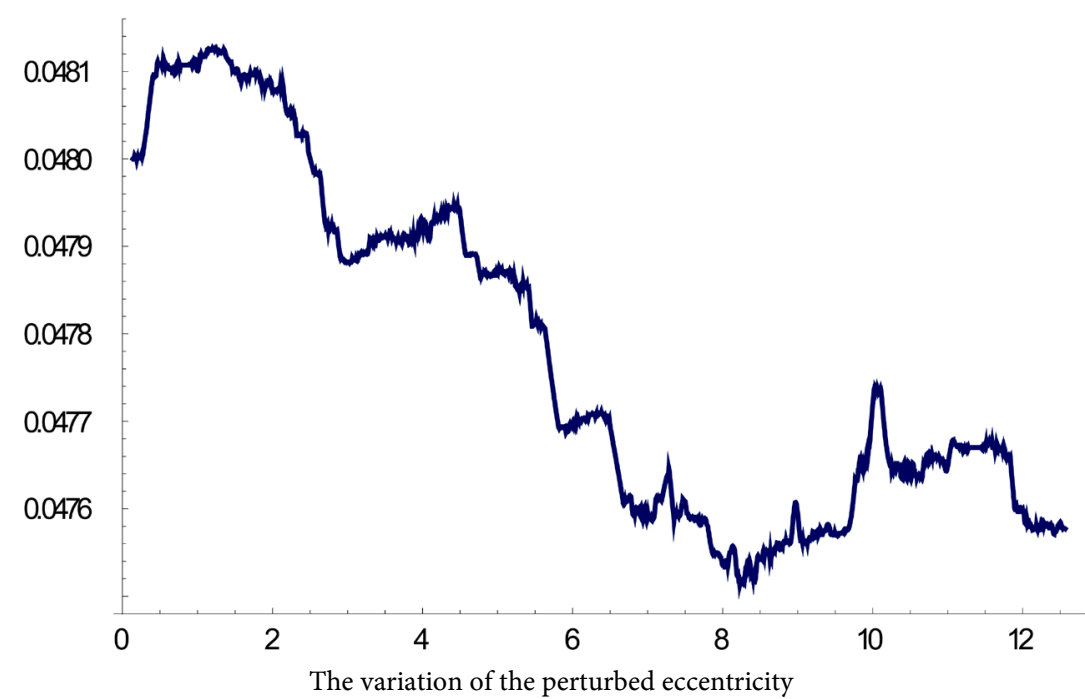

$\mathrm{X}$-axis represents the variation of time from 0 to $4 \pi$ in degree and $\mathrm{Y}$-axis represents $\delta \mathrm{e}$ in radian

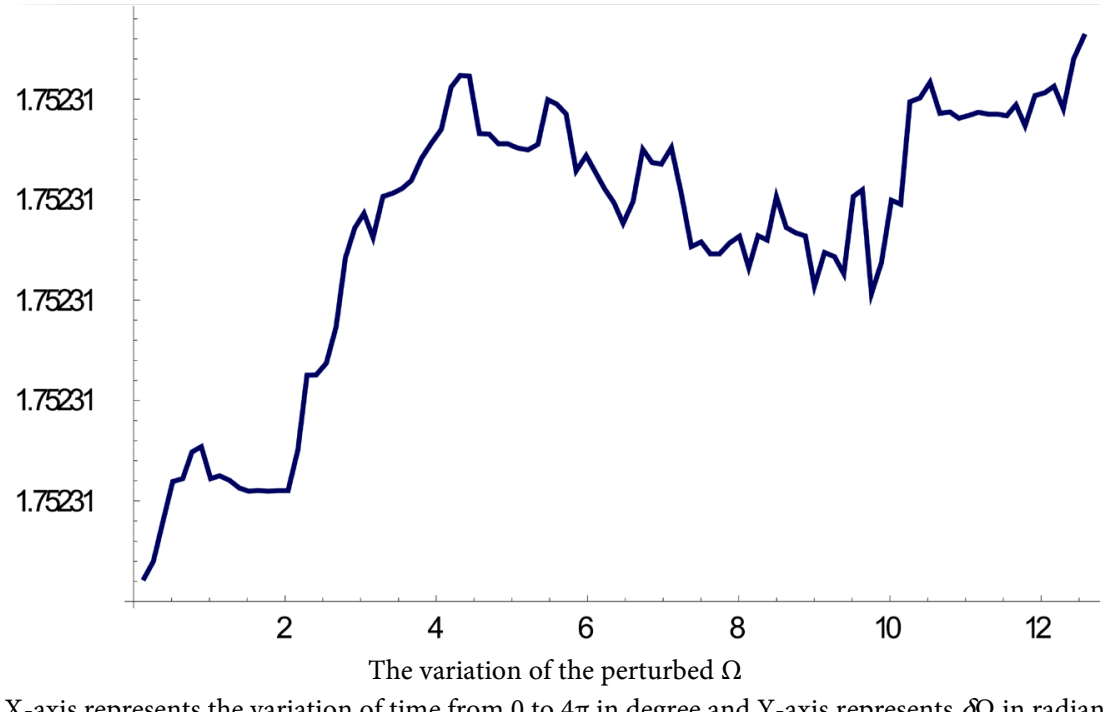




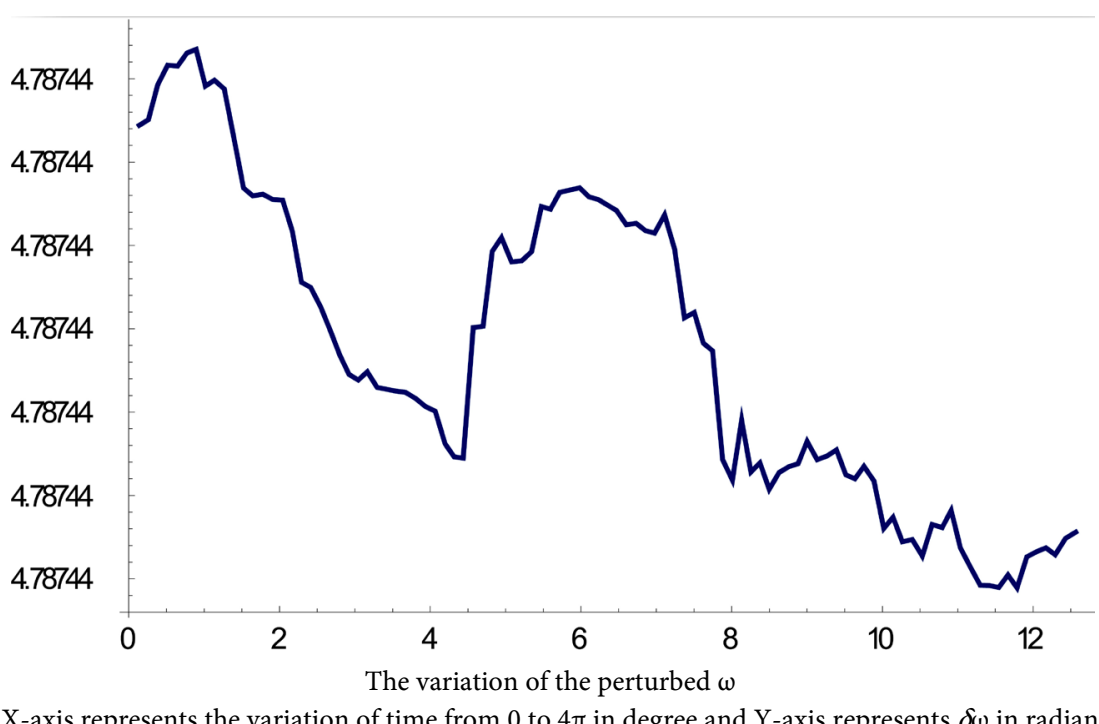

Figure 1. The short and long period perturbations of the orbital elements (semi-major, eccentricity, inclination, $\Omega, \omega)$ of Jupiter during the interval of time $\left(t-t_{0}\right)$, from 0 to $4 \pi$.

\section{Conclusion}

The resulting solution for our model from Equations (15) to (20) includes short-period as well as long-period terms for practical application it appears more expedient to calculate the perturbations on a revolution by revolution. The averaging process over the fast variable or short-period comes after the differentiated with respect to slow variable or long-period. The short-period perturbation on semi-major $a$, the eccentricity $e$, and the mean anomaly $M$ increase with each revolution during the time interval $\left(t-t_{0}\right)$ from zero to $4 \pi$. The long-period perturbation on the inclination $i, \Omega$, and $\omega$ takes long time to change with revolution in the same interval as seen in Figure 1. This paper provides a good approach for long-period orbital evolution studies for planets orbiting under the effect of the gravitational waves.

\section{References}

[1] Bertotti, B. (1973) Is the Solar System Gravitationally Closed? The Astrophysical Journal Letters, 14, 51.

[2] Rudenko, V.N. (1975) Test Bodies under the Effect of Gravitational Radiation. Soviet Astronomy Letters, 19, 270.

[3] Mashoon, B. (1978) On Tidal Resonance. Astrophysical Journal, 223, 285. https://doi.org/10.1086/156262

[4] Mashoon, B. (1987) Wave Propagation in a Gravitational Field. Physics Letters A, 122, 299-304.

[5] Turner, M.S. (1979) Influence of a Weak Gravitational Wave on a Bound System of Two Point-Masses. Astrophysical Journal, 233, 685. https://doi.org/10.1086/157429

[6] Nelson, L.A. and Chau, W.Y. (1982) Orbital Perturbations of a Gravitationally Bound Two-Body System with the Passage of Gravitational Waves. Astrophysical 
Journal, 254, 735. https://doi.org/10.1086/159785

[7] Ivanshchenko, A.V. (1987) The Variation of the Keplerian Elements of a Planetary Orbit under the Action of a Gravitational Wave. Soviet Astronomy Letters, 31, 76.

[8] Thorne, K.S. and Braginsky, V.B. (1976) Gravitational Wave Burst from the Nuclei of Distant Galaxies and Quasars. The Astrophysical Journal Letters, 204, L1. https://doi.org/10.1086/182042

[9] Lorenzo, I. (2014) Orbital Effect of a Monochromatic Plane GW with Ultra-Low Frequency Incident on a Gravitationally Bound Two-Body System. ScienceOpen Research, V2.

[10] Straumann, N. (1984) General Relativity and Relativistic Astrophysics. Springer-Verlag, Berlin. https://doi.org/10.1007/978-3-642-84439-3

[11] Smart, W.M. (1961) Celestial Mechanics. John Wiley, New York.

\section{Scientific Research Publishing}

Submit or recommend next manuscript to SCIRP and we will provide best service for you:

Accepting pre-submission inquiries through Email, Facebook, LinkedIn, Twitter, etc. A wide selection of journals (inclusive of 9 subjects, more than 200 journals)

Providing 24-hour high-quality service

User-friendly online submission system

Fair and swift peer-review system

Efficient typesetting and proofreading procedure

Display of the result of downloads and visits, as well as the number of cited articles

Maximum dissemination of your research work

Submit your manuscript at: http://papersubmission.scirp.org/

Or contactijaa@scirp.org 